\title{
On Semi-Tensor Bundle
}

\author{
Furkan Yildirım* \\ (Communicated by Arif Salimov)
}

\begin{abstract}
We investigate some lifts of tensor fields of type $(1,0)$ on a cross-section in the semi-tensor (pullback) bundle tM of tensor bundle TM of type $(p, q)$ by using projection (submersion) of the cotangent bundle $T^{*} M$ and we find some relation for them.
\end{abstract}

Keywords: Vector field; complete lift; cross-section; horizontal lift; pull-back bundle; cotangent bundle; semi-tensor bundle

AMS Subject Classification (2010): Primary: 53A45; Secondary: 55R10; 57R25.

\section{Introduction}

Let $M_{n}$ be an $n$-dimensional differentiable manifold of class $C^{\infty}$, and let $\left(T^{*}\left(M_{n}\right), \pi_{1}, M_{n}\right)$ be a cotangent bundle over $M_{n}$. We use the notation $\left(x^{i}\right)=\left(x^{\bar{\alpha}}, x^{\alpha}\right)$, where the indices $i, j, \ldots$ run from 1 to $2 n$, the indices $\bar{\alpha}, \bar{\beta}, \ldots$ from 1 to $n$ and the indices $\alpha, \beta, \ldots$ from $n+1$ to $2 n, x^{\alpha}$ are coordinates in $M_{n}, x^{\bar{\alpha}}=p_{\alpha}$ are fibre coordinates of the cotangent bundle $T^{*}\left(M_{n}\right)$.

Let now $\left(T_{q}^{p}\left(M_{n}\right), \tilde{\pi}, M_{n}\right)$ be a tensor bundle [3], [6], [[7], p.118] with base space $M_{n}$, and let $T^{*}\left(M_{n}\right)$ be cotangent bundle determined by a natural projection (submersion) $\pi_{1}: T^{*}\left(M_{n}\right) \rightarrow M_{n}$. The semi-tensor bundle (induced, pull-back [4],[5],[8],[9],[11],[12],,[13],[14]) of the tensor bundle $\left(T_{q}^{p}\left(M_{n}\right), \widetilde{\pi}, M_{n}\right)$ is the bundle $\left(t_{q}^{p}\left(M_{n}\right), \pi_{2}, T^{*}\left(M_{n}\right)\right)$ over cotangent bundle $T^{*}\left(M_{n}\right)$ with a total space

$$
\begin{aligned}
t_{q}^{p}\left(M_{n}\right) & =\left\{\left(\left(x^{\bar{\alpha}}, x^{\alpha}\right), x^{\overline{\bar{\alpha}}}\right) \in T^{*}\left(M_{n}\right) \times\left(T_{q}^{p}\right)_{x}\left(M_{n}\right): \pi_{1}\left(x^{\bar{\alpha}}, x^{\alpha}\right)=\widetilde{\pi}\left(x^{\alpha}, x^{\bar{\alpha}}\right)=\left(x^{\alpha}\right)\right\} \\
& \subset T^{*}\left(M_{n}\right) \times\left(T_{q}^{p}\right)_{x}\left(M_{n}\right)
\end{aligned}
$$

and with the projection map $\pi_{2}: t_{q}^{p}\left(M_{n}\right) \rightarrow T^{*}\left(M_{n}\right)$ defined by $\pi_{2}\left(x^{\bar{\alpha}}, x^{\alpha}, x^{\overline{\bar{\alpha}}}\right)=\left(x^{\bar{\alpha}}, x^{\alpha}\right)$, where $\left(T_{q}^{p}\right)_{x}\left(M_{n}\right)\left(x=\pi_{1}(\widetilde{x}), \widetilde{x}=\left(x^{\bar{\alpha}}, x^{\alpha}\right) \in T^{*}\left(M_{n}\right)\right)$ is the tensor space at a point $x$ of $M_{n}$, where $x^{\overline{\bar{\alpha}}}=t_{\alpha_{1} \ldots \alpha_{q}}^{\beta_{1} \ldots \beta_{p}}$ $\left(\overline{\bar{\alpha}}, \overline{\bar{\beta}}, \ldots=2 n+1, \ldots, 2 n+n^{p+q}\right)$ are fiber coordinates of the tensor bundle $T_{q}^{p}\left(M_{n}\right)$.

If $\left(x^{i^{\prime}}\right)=\left(x^{\bar{\alpha}^{\prime}}, x^{\alpha^{\prime}}, x^{\overline{\bar{\alpha}}^{\prime}}\right)$ is another system of local adapted coordinates in the semi-tensor bundle $t_{q}^{p}\left(M_{n}\right)$, then we have

$$
\left\{\begin{array}{l}
x^{\bar{\alpha}^{\prime}}=\frac{\partial x^{\beta}}{\partial x^{\prime}} p_{\beta}, \\
x^{\alpha^{\prime}}=x^{\alpha^{\prime}}\left(x^{\beta}\right), \\
x^{\overline{\bar{\alpha}}^{\prime}}=t_{\alpha_{1}^{\prime} \ldots \alpha_{q}^{\prime}}^{\beta_{1}^{\prime} \ldots \beta^{\prime}}=A_{\alpha_{1} \ldots \alpha_{p}}^{\beta_{1}^{\prime} \ldots \beta_{p}^{\prime}} A_{\alpha_{1}^{\prime} \ldots \alpha_{q}^{\prime}}^{\beta_{1} \ldots \beta_{q}} t_{\beta_{1} \ldots \beta_{q}}^{\alpha_{1} \ldots \alpha_{p}}=A_{(\alpha)}^{\left(\beta^{\prime}\right)} A_{\left(\alpha^{\prime}\right)}^{(\beta)} x^{\bar{\beta}} .
\end{array}\right.
$$

The Jacobian of (1.1) has the components

$$
\bar{A}=\left(A_{J}^{I^{\prime}}\right)=\left(\begin{array}{ccc}
A_{\alpha^{\prime}}^{\beta} & p_{\sigma} A_{\beta}^{\beta^{\prime}} A_{\beta^{\prime} \alpha^{\prime}}^{\sigma} & 0 \\
0 & A_{\beta}^{\alpha^{\prime}} & 0 \\
0 & t_{(\sigma)}^{(\alpha)} \partial_{\beta} A_{(\alpha)}^{\left(\beta^{\prime}\right)} A_{\left(\alpha^{\prime}\right)}^{(\sigma)} & A_{(\alpha)}^{\left(\beta^{\prime}\right)} A_{\left(\alpha^{\prime}\right)}^{(\beta)}
\end{array}\right),
$$

where $I=(\bar{\alpha}, \alpha, \overline{\bar{\alpha}}), J=(\bar{\beta}, \beta, \overline{\bar{\beta}}), I, J \ldots=1, \ldots, 2 n+n^{p+q}, t_{(\sigma)}^{(\alpha)}=t_{\sigma_{1} \ldots \sigma_{q}}^{\alpha_{1} \ldots \alpha_{p}}, A_{\beta}^{\alpha^{\prime}}=\frac{\partial x^{\alpha^{\prime}}}{\partial x^{\beta}}, A_{\alpha^{\prime}}^{\beta}=\frac{\partial x^{\beta}}{\partial x^{\alpha^{\prime}}}, A_{\beta^{\prime} \alpha^{\prime}}^{\sigma}=\frac{\partial^{2} x^{\sigma}}{\partial x^{\beta^{\prime}} \partial x^{\alpha^{\prime}}}$. It is easily verified that the condition $\operatorname{Det} \bar{A} \neq 0$ is equivalent to the condition:

$$
\operatorname{Det}\left(A_{\alpha^{\prime}}^{\beta}\right) \neq 0, \operatorname{Det}\left(A_{\beta}^{\alpha^{\prime}}\right) \neq 0, \operatorname{Det}\left(A_{(\alpha)}^{\left(\beta^{\prime}\right)} A_{\left(\alpha^{\prime}\right)}^{(\beta)}\right) \neq 0 .
$$


Also, $\operatorname{dim} t_{q}^{p}\left(M_{n}\right)=2 n+n^{p+q}$.

We note that special class of semi-tensor bundle was examined in [2]. The main purpose of this paper is to study semi-tensor (pull-back) bundle $t_{q}^{p}\left(M_{n}\right)$ of tensor bundle $T_{q}^{p}\left(M_{n}\right)$ by using projection of the cotangent bundle $T^{*}\left(M_{n}\right)$.

We denote by $\Im_{q}^{p}\left(T^{*}\left(M_{n}\right)\right)$ and $\Im_{q}^{p}\left(M_{n}\right)$ the modules over $F\left(T^{*}\left(M_{n}\right)\right)$ and $F\left(M_{n}\right)$ of all tensor fields of type $(p, q)$ on $T^{*}\left(M_{n}\right)$ and $M_{n}$, respectively, where $F\left(T^{*}\left(M_{n}\right)\right)$ and $F\left(M_{n}\right)$ denote the rings of real-valued $C^{\infty}$-functions on $T^{*}\left(M_{n}\right)$ and $M_{n}$, respectively.

\section{Vertical lifts of tensor fields and $\gamma$ - operator}

Let $A \in \Im_{q}^{p}\left(T^{*}\left(M_{n}\right)\right)$. On putting

$$
{ }^{v v} A=\left(\begin{array}{l}
v v A^{\bar{\alpha}} \\
{ }^{v v} A^{\alpha} \\
{ }^{v v} A^{\bar{\alpha}}
\end{array}\right)=\left(\begin{array}{l}
0 \\
0 \\
A_{\beta_{1} \ldots \beta_{q}}^{\alpha_{1} \ldots \alpha_{p}}
\end{array}\right),
$$

from (1.2), we easily see that with ${ }^{v v} A^{\prime}=\bar{A}\left({ }^{v v} A\right)$. The vector field ${ }^{v v} A \in \Im_{0}^{1}\left(t_{q}^{p}\left(M_{n}\right)\right)$ is called the vertical lift of $A \in \Im_{q}^{p}\left(T^{*}\left(M_{n}\right)\right)$ to the semi-tensor bundle $t_{q}^{p}\left(M_{n}\right)$.

Let $\varphi \in \Im_{1}^{1}\left(M_{n}\right)$. We define a vector field $\gamma \varphi$ in $\pi^{-1}(U)$ by

$$
\begin{cases}\gamma \varphi=\left(\sum_{\lambda=1}^{p} t_{\beta_{1} \ldots \beta_{q}}^{\alpha_{1} \ldots \alpha_{p}} \varphi_{\varepsilon}^{\alpha_{\lambda}}\right) \frac{\partial}{\partial x^{\bar{\beta}}}, & (p \geq 1, q \geq 0) \\ \widetilde{\gamma} \varphi=\left(\sum_{\mu=1}^{q} t_{\beta_{1} \ldots \varepsilon \ldots \beta_{q}}^{\alpha_{1} \ldots \alpha_{p}} \varphi_{\beta_{\mu}}^{\varepsilon}\right) \frac{\partial}{\partial x^{\overline{\bar{\beta}}}}, & (p \geq 0, q \geq 1)\end{cases}
$$

with respect to the coordinates $\left(x^{\bar{\beta}}, x^{\beta}, x^{\overline{\bar{\beta}}}\right)$ on $t_{q}^{p}\left(M_{n}\right)$. From (1.2) we easily see that the vector fields $\gamma \varphi$ and $\widetilde{\gamma} \varphi$ defined in each $\pi^{-1}(U) \subset t_{q}^{p}\left(M_{n}\right)$ determine respectively global vertical vector fields on $t_{q}^{p}\left(M_{n}\right)$. We call $\gamma \varphi$ (or $\widetilde{\gamma} \varphi)$ the vertical-vector lift of the tensor field $\varphi \in \Im_{1}^{1}\left(M_{n}\right)$ to $t_{q}^{p}\left(M_{n}\right)$. For any $\varphi \in \Im_{1}^{1}\left(M_{n}\right)$, if we take account of (1.2) and (2.2), we can prove that $(\gamma \varphi)^{\prime}=\bar{A}(\gamma \varphi)$. Where $\gamma \varphi$ is a vector field defined by

$$
\gamma \varphi=(\gamma \varphi)^{I}=\left(\begin{array}{l}
0 \\
0 \\
\sum_{\lambda=1}^{p} t_{\beta_{1} \ldots \beta_{q}}^{\alpha_{1} \ldots \alpha_{p}} \varphi_{\varepsilon}^{\alpha_{\lambda}}
\end{array}\right) .
$$

Let $\varphi \in \Im_{1}^{1}\left(M_{n}\right)$. On putting

$$
\tilde{\gamma} \varphi=(\widetilde{\gamma} \varphi)^{I}=\left(\begin{array}{l}
0 \\
0 \\
\sum_{\mu=1}^{q} t_{\beta_{1} \ldots \varepsilon \ldots \beta_{q}}^{\alpha_{1} \ldots \alpha_{p}} \varphi_{\beta_{\mu}}^{\varepsilon}
\end{array}\right),
$$

we easily see that $(\widetilde{\gamma} \varphi)^{\prime}=\bar{A}(\widetilde{\gamma} \varphi)$.

For any $\varphi \in \Im_{1}^{1}\left(T^{*}\left(M_{n}\right)\right)$, if we take account of (1.2), we can prove that $(\gamma \varphi)^{\prime}=\bar{A}(\gamma \varphi)$, where $\gamma \varphi$ is a vector field defined by

$$
\gamma \varphi=\left(\begin{array}{l}
-p_{\sigma} F_{\beta}^{\sigma} \\
0 \\
0
\end{array}\right)
$$

with respect to the coordinates $\left(x^{\bar{\beta}}, x^{\beta}, x^{\bar{\beta}}\right)$.

\section{Complete lifts of vector fields}

Let $X \in \Im_{0}^{1}\left(T^{*}\left(M_{n}\right)\right)$, i.e. $X=X^{\alpha} \partial_{\alpha}$. The complete lift ${ }^{c} X$ of $X$ to cotangent bundle is defined by ${ }^{c} X=$ $X^{\alpha} \partial_{\alpha}-p_{\beta}\left(\partial_{\alpha} X^{\beta}\right) \partial_{\bar{\alpha}}[[10]$, p.236]. On putting

$$
{ }^{c c} X=\left(\begin{array}{l}
{ }^{c c} X^{\bar{\beta}} \\
{ }^{c c} X^{\beta} \\
{ }^{c c} X^{\bar{\beta}}
\end{array}\right)=\left(\begin{array}{l}
-p_{\varepsilon}\left(\partial_{\beta} X^{\varepsilon}\right) \\
X^{\beta} \\
\sum_{\lambda=1}^{p} t_{\beta_{1} \ldots \beta_{q}}^{\alpha_{1} \ldots \alpha_{p}} \partial_{\varepsilon} X^{\alpha_{\lambda}}-\sum_{\mu=1}^{q} t_{\beta_{1} \ldots \varepsilon \ldots \beta_{q}}^{\alpha_{1} \ldots \alpha_{p}} \partial_{\beta_{\mu}} X^{\varepsilon}
\end{array}\right),
$$

from (1.2), we easily see that ${ }^{c c} X^{\prime}=\bar{A}\left({ }^{c c} X\right)$. The vector field ${ }^{c c} X$ is called the complete lift of ${ }^{c} X \in \Im_{0}^{1}\left(T^{*}\left(M_{n}\right)\right)$ to $t_{q}^{p}\left(M_{n}\right)$. 


\section{Horizontal lifts of vector fields}

Let $X \in \Im_{0}^{1}\left(T^{*}\left(M_{n}\right)\right)$, i.e. $X=X^{\alpha}\left(x^{\alpha}\right) \partial_{\alpha}$. If we take account of (1.2), we can prove that ${ }^{H H} X^{\prime}=\bar{A}\left({ }^{H H} X\right)$, where ${ }^{H H} X \in \Im_{0}^{1}\left(t_{q}^{p}\left(M_{n}\right)\right)$ is a vector field defined by

$$
{ }^{H H} X=\left(\begin{array}{l}
X^{\alpha} \Gamma_{\alpha \beta} \\
X^{\beta} \\
X^{l}\left(\sum_{\mu=1}^{q} \Gamma_{l \beta_{\mu}}^{\varepsilon} t_{\beta_{1} \ldots \varepsilon \ldots \beta_{q}}^{\alpha_{1} \ldots \alpha_{p}}-\sum_{\lambda=1}^{p} \Gamma_{l \varepsilon}^{\alpha_{\lambda}} t_{\beta_{1} \ldots \beta_{q}}^{\alpha_{1} \ldots \alpha_{p}}\right)
\end{array}\right),
$$

with respect to the coordinates $\left(x^{\bar{\beta}}, x^{\beta}, x^{\overline{\bar{\beta}}}\right)$ on $t_{q}^{p}\left(M_{n}\right)$. We call ${ }^{H}{ }^{H} X$ the horizontal lift of the vector field $X$ to $t_{q}^{p}\left(M_{n}\right)$, where $\Gamma_{\alpha \beta}=p_{\varepsilon} \Gamma_{\alpha \beta}^{\varepsilon}$.

Theorem 4.1. If $X \in \Im_{0}^{1}\left(T^{*}\left(M_{n}\right)\right)$ then

$$
{ }^{c c} X-{ }^{H H} X=\gamma(\hat{\nabla} X)-\widetilde{\gamma}(\hat{\nabla} X)+\gamma(\nabla X),
$$

where the symmetric affine connection $\hat{\nabla}$ is the given by $\widehat{\Gamma}_{\beta \theta}^{\alpha}=\Gamma_{\theta \beta}^{\alpha}$.

Proof. From (2.3), (2.4), (2.5), (3.1) and (4.1), we have

$$
\begin{aligned}
& { }^{c c} X-{ }^{H H} X=\left(\begin{array}{l}
-p_{\varepsilon}\left(\partial_{\beta} X^{\varepsilon}\right) \\
X^{\beta} \\
\sum_{\lambda=1}^{p} t_{\beta_{1} \ldots \beta_{q}}^{\alpha_{1} \ldots \alpha_{p}} \partial_{\varepsilon} X^{\alpha_{\lambda}}-\sum_{\mu=1}^{q} t_{\beta_{1} \ldots \varepsilon \varepsilon \beta_{q}}^{\alpha_{1} \ldots \alpha_{p}} \partial_{\beta_{\mu}} X^{\varepsilon}
\end{array}\right) \\
& -\left(\begin{array}{l}
p_{\varepsilon} X^{\alpha} \Gamma_{\alpha \beta}^{\varepsilon} \\
X^{\beta} \\
X^{l}\left(\sum_{\mu=1}^{q} \Gamma_{l \beta_{\mu}}^{\varepsilon} t_{\beta_{1} \ldots \varepsilon \ldots \beta_{q}}^{\alpha_{1} \ldots \alpha_{p}}-\sum_{\lambda=1}^{p} \Gamma_{l \varepsilon}^{\alpha_{\lambda}} t_{\beta_{1} \ldots \beta_{q}}^{\alpha_{1} \ldots \alpha_{p}}\right)
\end{array}\right) \\
& =\left(\begin{array}{l}
-p_{\varepsilon}\left(\partial_{\beta} X^{\varepsilon}\right)-p_{\varepsilon} X^{\alpha} \Gamma_{\alpha \beta}^{\varepsilon} \\
0 \\
\sum_{\lambda=1}^{p} t_{\beta_{1} \ldots \beta_{q}}^{\alpha_{1} \ldots \varepsilon \alpha_{p}}\left(\partial_{\varepsilon} X^{\alpha_{\lambda}}+\Gamma_{l \varepsilon}^{\alpha_{\lambda}} X^{l}\right)-\sum_{\mu=1}^{q} t_{\beta_{1} \ldots \varepsilon \varepsilon \beta_{q}}^{\alpha_{1} \ldots \alpha_{p}}\left(\partial_{\beta_{\mu}} X^{\varepsilon}+\Gamma_{l \beta_{\mu}}^{\varepsilon} X^{l}\right)
\end{array}\right) \\
& =\left(\begin{array}{l}
0 \\
0 \\
\sum_{\lambda=1}^{p} t_{\beta_{1} \ldots \beta_{q}}^{\alpha_{1} \ldots \alpha_{p}}\left(\partial_{\varepsilon} X^{\alpha_{\lambda}}+\Gamma_{l \varepsilon}^{\alpha_{\lambda}} X^{l}\right)
\end{array}\right)-\left(\begin{array}{l}
0 \\
0 \\
\sum_{\mu=1}^{q} t_{\beta_{1} \ldots \varepsilon \ldots \beta_{q}}^{\alpha_{1} \ldots \alpha_{p}}\left(\partial_{\beta_{\mu}} X^{\varepsilon}+\Gamma_{l \beta_{\mu}}^{\varepsilon} X^{l}\right)
\end{array}\right) \\
& +\left(\begin{array}{l}
-p_{\varepsilon}\left(\partial_{\beta} X^{\varepsilon}+X^{\alpha} \Gamma_{\alpha \beta}^{\varepsilon}\right) \\
0
\end{array}\right) \\
& =\left(\begin{array}{l}
0 \\
\sum_{\lambda=1}^{p} t_{\beta_{1} \ldots \beta_{q}}^{\alpha_{1} \ldots \varepsilon \alpha_{p}}(\underbrace{\partial_{\varepsilon} X^{\alpha_{\lambda}}+\widehat{\Gamma}_{\varepsilon l}^{\alpha_{\lambda}} X^{l}}_{\hat{\nabla}_{\varepsilon} \widetilde{X}^{\alpha_{\lambda}}})
\end{array}\right)-\left(\begin{array}{l}
0 \\
0 \\
\sum_{\mu=1}^{q} t_{\beta_{1} \ldots \varepsilon \ldots \beta_{q}}^{\alpha_{1} \ldots \alpha_{p}}(\underbrace{\partial_{\beta_{\mu}} X^{\varepsilon}+\widehat{\Gamma}_{\beta_{\mu} l}^{\varepsilon} X^{l}}_{\hat{\nabla}_{\beta_{\mu}} \widetilde{X}^{\varepsilon}})
\end{array}\right) \\
& +\left(\begin{array}{l}
-p_{\varepsilon}(\underbrace{\partial_{\beta} X^{\varepsilon}+X^{\alpha} \Gamma_{\alpha \beta}^{\varepsilon}}_{\nabla_{\beta} X^{\varepsilon}}) \\
0 \\
0
\end{array}\right) \\
& =\left(\begin{array}{l}
0 \\
0 \\
\sum_{\lambda=1}^{p} t_{\beta_{1} \ldots \beta_{q}}^{\alpha_{1} \ldots \alpha_{p}}\left(\hat{\nabla}_{\varepsilon} \widetilde{X}^{\alpha_{\lambda}}\right)
\end{array}\right)-\left(\begin{array}{l}
0 \\
0 \\
\sum_{\mu=1}^{q} t_{\beta_{1} \ldots \varepsilon \ldots \beta_{q}}^{\alpha_{1} \ldots \alpha_{p}}\left(\hat{\nabla}_{\beta_{\mu}} \widetilde{X}^{\varepsilon}\right)
\end{array}\right)+\left(\begin{array}{l}
-p_{\varepsilon}\left(\nabla_{\beta} X^{\varepsilon}\right) \\
0 \\
0
\end{array}\right) \\
& =\gamma\left(\hat{\nabla}_{\varepsilon} \widetilde{X}^{\alpha_{\lambda}}\right)-\widetilde{\gamma}\left(\hat{\nabla}_{\beta_{\mu}} \widetilde{X}^{\varepsilon}\right)+\gamma\left(\nabla_{\beta} X^{\varepsilon}\right)=\gamma(\hat{\nabla} X)-\widetilde{\gamma}(\hat{\nabla} X)+\gamma(\nabla X) \text {, }
\end{aligned}
$$

which prove Theorem 4.1. 


\section{Cross-sections in the semi-tensor bundle}

Let $\xi \in \Im_{q}^{p}\left(M_{n}\right)$ be a tensor field on $M_{n}$. Then the correspondence $x \rightarrow \xi_{x}$, $\xi_{x}$ being the value of $\xi$ at $x \in T^{*}\left(M_{n}\right)$, determines a cross-section $\beta_{\xi}$ of semi-tensor bundle. Thus if $\sigma_{\xi}: M_{n} \rightarrow T_{q}^{p}\left(M_{n}\right)$ is a cross-section of $\left(T_{q}^{p}\left(M_{n}\right), \tilde{\pi}, M_{n}\right)$, such that $\tilde{\pi} \circ \sigma_{\xi}=I_{\left(M_{n}\right)}$, an associated cross-section $\beta_{\xi}: T^{*}\left(M_{n}\right) \rightarrow t_{q}^{p}\left(M_{n}\right)$ of semi-tensor bundle $\left(t_{q}^{p}\left(M_{n}\right), \pi_{2}, T^{*}\left(M_{n}\right)\right)$ defined by [[1], p. 217-218], [4], [5], [[10].p. 301]:

$$
\beta_{\xi}\left(x^{\bar{\alpha}}, x^{\alpha}\right)=\left(x^{\bar{\alpha}}, x^{\alpha}, \sigma_{\xi} \circ \pi_{1}\left(x^{\bar{\alpha}}, x^{\alpha}\right)\right)=\left(x^{\bar{\alpha}}, x^{\alpha}, \sigma_{\xi}\left(x^{\alpha}\right)\right)=\left(x^{\bar{\alpha}}, x^{\alpha}, \xi_{\beta_{1} \ldots \beta_{q}}^{\alpha_{1} \ldots \alpha_{p}}\left(x^{\beta}\right)\right) .
$$

If the tensor field $\xi$ has the local components $\xi_{\beta_{1} \ldots \beta_{q}}^{\alpha_{1} \ldots \alpha_{p}}\left(x^{\beta}\right)$, the cross-section $\beta_{\xi}\left(T^{*}\left(M_{n}\right)\right)$ of $t_{q}^{p}\left(M_{n}\right)$ is locally expressed by

$$
\left\{\begin{aligned}
x^{\bar{\beta}} & =p_{\beta}=\theta_{\beta}\left(x^{\alpha}\right), \\
x^{\beta} & =x^{\beta} \\
x^{\bar{\beta}} & =\xi_{\beta_{1} \ldots \beta_{q}}^{\alpha_{1} \ldots \alpha_{p}}\left(x^{\alpha}\right),
\end{aligned}\right.
$$

with respect to the coordinates $x^{B}=\left(x^{\bar{\beta}}, x^{\beta}, x^{\bar{\beta}}\right)$ in $t_{q}^{p}\left(M_{n}\right)$.

$x^{\bar{\alpha}}=p_{\alpha}$ being considered as parameters. Thus, by differentiating with respect to $p_{\alpha}$, we easily see that the $n$ local vector fields $B_{(\bar{\theta})}(\bar{\theta}=1, \ldots, n)$ with components

$$
B_{(\bar{\theta})}:\left(B_{(\bar{\theta})}^{B}\right)=\partial_{(\bar{\theta})} x^{B}=\left(\begin{array}{l}
\partial_{\bar{\theta}} \theta_{\beta} \\
\partial_{\bar{\theta}} x^{\beta} \\
\partial_{\bar{\theta}} \xi_{\beta_{1} \ldots \beta_{q}}^{\alpha_{1} \ldots \alpha_{p}}
\end{array}\right)=\left(\begin{array}{l}
\delta_{\beta}^{\theta} \\
0 \\
0
\end{array}\right)
$$

is tangent to the fibre, where

$$
\delta_{\beta}^{\theta}=A_{\beta}^{\theta}=\frac{\partial x^{\theta}}{\partial x^{\beta}} .
$$

Let $\omega$ be an 1-form with local components $\omega_{\beta}$ on $M_{n}$, so that $\omega$ is a 1-form with local expression $\omega=\omega_{\beta} d x^{\beta}$. We denote by $B \omega$ the vector field with local components

$$
B \omega:\left(B_{(\bar{\theta})}^{B} \omega_{\theta}\right)=\left(\begin{array}{l}
\omega_{\beta} \\
0 \\
0
\end{array}\right)
$$

which is tangent to the fibre.

Taking the derivative with respect to $x^{\theta}$, we have vector fields $C_{(\theta)}(\theta=n+1, \ldots, 2 n)$ with components

$$
C_{(\theta)}=\frac{\partial x^{B}}{\partial x^{\theta}}=\partial_{\theta} x^{B}=\left(\begin{array}{l}
\partial_{\theta} \theta_{\beta} \\
\partial_{\theta} x^{\beta} \\
\partial_{\theta} \xi_{\beta_{1} \ldots \beta_{q}}^{\alpha_{1} \ldots \alpha_{p}}
\end{array}\right),
$$

which are tangent to the cross-section $\beta_{\xi}\left(T^{*}\left(M_{n}\right)\right)$.

Thus $C_{(\theta)}$ has the components

$$
C_{(\theta)}:\left(C_{(\theta)}^{B}\right)=\left(\begin{array}{l}
\partial_{\theta} \theta_{\beta} \\
\delta_{\theta}^{\beta} \\
\partial_{\theta} \xi_{\beta_{1} \ldots \beta_{q}}^{\alpha_{1} \ldots \alpha_{p}}
\end{array}\right),
$$

with respect to the coordinates $\left(x^{\bar{\beta}}, x^{\beta}, x^{\bar{\beta}}\right)$ in $t_{q}^{p}\left(M_{n}\right)$. Where

$$
\delta_{\theta}^{\beta}=A_{\theta}^{\beta}=\frac{\partial x^{\beta}}{\partial x^{\theta}} .
$$

Let $X \in \Im_{0}^{1}\left(T^{*}\left(M_{n}\right)\right)$. Then we denote by $C X$ the vector field with local components

$$
C X:\left(C_{(\theta)}^{B} X^{\theta}\right)=\left(\begin{array}{l}
X^{\theta} \partial_{\theta} \theta_{\beta} \\
X^{\beta} \\
X^{\theta} \partial_{\theta} \xi_{\beta_{1} \ldots \beta_{q}}^{\alpha_{1} \ldots \alpha_{p}}
\end{array}\right)
$$


with respect to the coordinates $\left(x^{\bar{\beta}}, x^{\beta}, x^{\bar{\beta}}\right)$ in $t_{q}^{p}\left(M_{n}\right)$, which is defined globally along $\beta_{\xi}\left(T^{*}\left(M_{n}\right)\right)$.

On the other hand, the fibre is locally expressed by

$$
\left\{\begin{array}{l}
x^{\bar{\beta}}=p_{\beta}=\text { const. } \\
x^{\beta}=\text { const. } \\
x^{\overline{\bar{\beta}}}=t_{\beta_{1} \ldots \beta_{q}}^{\alpha_{1} \ldots \alpha_{p}}=t_{\beta_{1} \ldots \beta_{q}}^{\alpha_{1} \ldots \alpha_{p}}
\end{array}\right.
$$

$t_{\beta_{1} \ldots \beta_{q}}^{\alpha_{1} \ldots \alpha_{p}}$ being considered as parameters. Thus, by differentiating with respect to $x^{\overline{\bar{\beta}}}=t_{\beta_{1} \ldots \beta_{q}}^{\alpha_{1} \ldots \alpha_{p}}$, we easily see that the vector fields $E_{(\bar{\theta})}\left(\bar{\theta}=2 n+1, \ldots, 2 n+n^{p+q}\right)$ with components

$$
E_{(\overline{\bar{\theta}})}:\left(E_{(\overline{\bar{\theta}})}^{B}\right)=\partial_{\overline{\bar{\theta}}} x^{B}=\left(\begin{array}{l}
\partial_{\overline{\bar{\theta}}} \theta_{\beta} \\
\partial_{\overline{\bar{\theta}}} x^{\beta} \\
\partial_{\overline{\bar{\theta}}} \bar{t}_{\beta_{1} \ldots \beta_{q}}^{\alpha_{1} \ldots \alpha_{p}}
\end{array}\right)=\left(\begin{array}{l}
0 \\
0 \\
\delta_{\beta_{1} \ldots \delta_{\beta_{q}}^{\theta_{1}} \theta_{\gamma_{1}}^{\theta_{q}} \ldots \delta_{\gamma_{p}}^{\alpha_{p}}}^{\alpha_{1}}
\end{array}\right)
$$

is tangent to the fibre, where $\delta$ is the Kronecker symbol.

Let $\xi$ be a tensor field of type $(p, q)$ with local components

$$
\xi=\xi_{\theta_{1} \ldots \theta_{q}}^{\gamma_{1} \ldots \gamma_{p}} d x^{\theta_{1}} \otimes \ldots \otimes d x^{\theta_{q}} \otimes \partial_{\gamma_{1}} \otimes \ldots \otimes \partial_{\gamma_{p}}
$$

on $M_{n}$.

We denote by $E \xi$ the vector field with local components

$$
E \xi:\left(E_{(\overline{\bar{\theta}})}^{B} \xi_{\theta_{1} \ldots \theta_{q}}^{\gamma_{1} \ldots \gamma_{p}}\right)=\left(\begin{array}{l}
0 \\
0 \\
\xi_{\beta_{1} \ldots \beta_{q}}^{\alpha_{1} \ldots \alpha_{p}}
\end{array}\right)
$$

which is tangent to the fibre.

Theorem 5.1. Let $\psi, \omega \in \Im_{1}^{0}\left(M_{n}\right)$. For the Lie product, we have

$$
[B \psi, B \omega]=0 .
$$

Proof. If $\psi, \omega \in \Im_{1}^{0}\left(M_{n}\right)$ and $\left(\begin{array}{c}{[B \psi, B \omega]^{\bar{\beta}}} \\ {[B \psi, B \omega]^{\beta}} \\ {[B \psi, B \omega]^{\bar{\beta}}}\end{array}\right)$ are the components of $[B \psi, B \omega]$ with respect to the coordinates $\left(x^{\bar{\beta}}, x^{\beta}, x^{\bar{\beta}}\right)$ in $t_{q}^{p}\left(M_{n}\right)$, then we have

$$
\begin{aligned}
{[B \psi, B \omega]^{J} } & =\psi^{I} \partial_{I} \omega^{J}-\omega^{I} \partial_{I} \psi^{J} \\
& =\psi^{\bar{\alpha}} \partial_{\bar{\alpha}} \omega^{J}+\psi^{\alpha} \partial_{\alpha} \omega^{J}+\psi^{\bar{\alpha}} \partial_{\overline{\bar{\alpha}}} \omega^{J}-\omega^{\bar{\alpha}} \partial_{\bar{\alpha}} \psi^{J}-\omega^{\alpha} \partial_{\alpha} \psi^{J}-\omega^{\bar{\alpha}} \partial_{\overline{\bar{\alpha}}} \psi^{J} \\
& =\psi_{\alpha} \partial_{\bar{\alpha}} \omega^{J}-\omega_{\alpha} \partial_{\bar{\alpha}} \psi^{J} .
\end{aligned}
$$

Firstly, if $J=\bar{\beta}$, we have

$$
\begin{aligned}
{[B \psi, B \omega]^{\bar{\beta}} } & =\psi_{\alpha} \partial_{\bar{\alpha}} \omega^{\bar{\beta}}-\omega_{\alpha} \partial_{\bar{\alpha}} \psi^{\bar{\beta}} \\
& =\psi_{\alpha} \partial_{\bar{\alpha}} \omega_{\beta}-\omega_{\alpha} \partial_{\bar{\alpha}} \psi_{\beta} \\
& =0
\end{aligned}
$$

by virtue of (5.2). Secondly, if $J=\beta$, we have

$$
\begin{aligned}
{[B \psi, B \omega]^{\beta} } & =\psi_{\alpha} \partial_{\bar{\alpha}} \omega^{\beta}-\omega_{\alpha} \partial_{\bar{\alpha}} \psi^{\beta} \\
& =0
\end{aligned}
$$

by virtue of (5.2). Thirdly, if $J=\overline{\bar{\beta}}$. Then we have

$$
\begin{aligned}
{[B \psi, B \omega]^{\overline{\bar{\beta}}} } & =\psi_{\alpha} \partial_{\bar{\alpha}} \omega^{\overline{\bar{\beta}}}-\omega_{\alpha} \partial_{\bar{\alpha}} \psi^{\overline{\bar{\beta}}} \\
& =0
\end{aligned}
$$

by virtue of (5.2). Thus, we have $[B \psi, B \omega]=0$. 
Theorem 5.2. Let $X$ be a vector field on $T^{*}\left(M_{n}\right)$, we have along $\beta_{\xi}\left(T^{*}\left(M_{n}\right)\right)$ the formula

$$
{ }^{c c} X=-B\left(L_{X} \theta\right)+C X+E\left(-L_{X} \xi\right),
$$

where $L_{X} \theta$ denotes the Lie derivative of $\theta$ with respect to $X$, and $L_{X} \xi$ denotes the Lie derivative of $\xi$ with respect to $X$. Proof. Using (3.1), (5.2), (5.3) and (5.4), we have

$$
\begin{aligned}
& -B\left(L_{X} \theta\right)+C X+E\left(-L_{X} \xi\right)=-\left(\begin{array}{l}
X^{\theta} \partial_{\theta} \theta_{\beta}+\theta_{\theta} \partial_{\beta} X^{\theta} \\
0 \\
0
\end{array}\right)+\left(\begin{array}{l}
X^{\theta} \partial_{\theta} \theta_{\beta} \\
X^{\beta} \\
X^{\theta} \partial_{\theta} \xi_{\beta_{1} \ldots \beta_{q}}^{\alpha_{1} \ldots \alpha_{p}}
\end{array}\right) \\
& +\left(\begin{array}{l}
0 \\
0 \\
-X^{\theta} \partial_{\theta} \xi_{\beta_{1} \ldots \beta_{q}}^{\alpha_{1} \ldots \alpha_{p}}-\sum_{\mu=1}^{q} \partial_{\beta_{\mu}} X^{\beta} \xi_{\beta_{1} \ldots \beta \ldots \beta_{q}}^{\alpha_{1} \ldots \alpha_{p}}+\sum_{\lambda=1}^{p} \partial_{\beta} X^{\alpha_{\lambda}} \xi_{\beta_{1} \ldots \beta_{q}}^{\alpha_{1} \ldots \varepsilon \ldots \alpha_{p}}
\end{array}\right) \\
& =\left(\begin{array}{l}
-\theta_{\theta}\left(\partial_{\beta} X^{\theta}\right) \\
X^{\beta} \\
-\sum_{\mu=1}^{q} \partial_{\beta_{\mu}} X^{\beta} \xi_{\beta_{1} \ldots \beta \ldots \beta_{q}}^{\alpha_{1} \ldots \alpha_{p}}+\sum_{\lambda=1}^{p} \partial_{\beta} X^{\alpha_{\lambda}} \xi_{\beta_{1} \ldots \beta_{q}}^{\alpha_{1} \ldots \varepsilon \ldots \alpha_{p}}
\end{array}\right) \\
& ={ }^{c c} X \text {. }
\end{aligned}
$$

Thus, we have Theorem 5.2.

On the other hand, on putting $C_{(\overline{\bar{\beta}})}=E_{(\overline{\bar{\beta}})}$, we write the adapted frame of $\beta_{\xi}\left(T^{*}\left(M_{n}\right)\right)$ as $\left\{B_{(\bar{\beta})}, C_{(\beta)}, C_{(\bar{\beta})}\right\}$. The adapted frame $\left\{B_{(\bar{\beta})}, C_{(\beta)}, C_{(\bar{\beta})}\right\}$ of $\beta_{\xi}\left(T^{*}\left(M_{n}\right)\right)$ is given by the matrix

$$
\widetilde{A}=\left(\widetilde{A}_{B}^{A}\right)=\left(\begin{array}{ccc}
\delta_{\alpha}^{\beta} & \partial_{\beta} \theta_{\alpha} & 0 \\
0 & \delta_{\beta}^{\alpha} & 0 \\
0 & \partial_{\beta} \xi_{\alpha_{1} \ldots \alpha_{q}}^{\sigma_{1} \ldots \sigma_{p}} & \delta_{\alpha_{1}}^{\beta_{1}} \ldots \delta_{\alpha_{q}}^{\beta_{q}} \delta_{\gamma_{1}}^{\sigma_{1}} \ldots \delta_{\gamma_{p}}^{\sigma_{p}}
\end{array}\right)
$$

Since the matrix $\widetilde{A}$ in (5.5) is non-singular, it has the inverse. Denoting this inverse by $(\widetilde{A})^{-1}$, we have

$$
(\widetilde{A})^{-1}=\left(\widetilde{A}_{C}^{B}\right)^{-1}=\left(\begin{array}{ccc}
\delta_{\beta}^{\theta} & -\partial_{\theta} \theta_{\beta} & 0 \\
0 & \delta_{\theta}^{\beta} & 0 \\
0 & -\partial_{\theta} \xi_{\beta_{1} \ldots \beta_{q}}^{\sigma_{1} \ldots \sigma_{p}} & \delta_{\beta_{1}}^{\theta_{1}} \ldots \delta_{\beta_{q}}^{\theta_{q}} \delta_{\gamma_{1}}^{\sigma_{1}} \ldots \delta_{\gamma_{q}}^{\sigma_{p}}
\end{array}\right)
$$

where $\widetilde{A}(\widetilde{A})^{-1}=\left(\widetilde{A}_{B}^{A}\right)\left(\widetilde{A}_{C}^{B}\right)^{-1}=\delta_{C}^{A}=\widetilde{I}$, where $A=(\bar{\alpha}, \alpha, \overline{\bar{\alpha}}), B=(\bar{\beta}, \beta, \overline{\bar{\beta}}), C=(\bar{\theta}, \theta, \overline{\bar{\theta}})$.

Proof. In fact, from (5.5) and (5.6), we easily see that

$$
\begin{aligned}
& \widetilde{A}(\widetilde{A})^{-1}=\left(\widetilde{A}_{B}^{A}\right)\left(\widetilde{A}_{C}^{B}\right)^{-1} \\
& =\left(\begin{array}{ccc}
\delta_{\alpha}^{\beta} & \partial_{\beta} \theta_{\alpha} & 0 \\
0 & \delta_{\beta}^{\alpha} & 0 \\
0 & \partial_{\beta} \xi_{\alpha_{1} \ldots \alpha_{q}}^{\sigma_{1} \ldots \sigma_{p}} & \delta_{\alpha_{1}}^{\beta_{1}} \ldots \delta_{\alpha_{q}}^{\beta_{q}} \delta_{\gamma_{1}}^{\sigma_{1}} \ldots \delta_{\gamma_{p}}^{\sigma_{p}}
\end{array}\right)\left(\begin{array}{ccc}
\delta_{\beta}^{\theta} & -\partial_{\theta} \theta_{\beta} & 0 \\
0 & \delta_{\theta}^{\beta} & 0 \\
0 & -\partial_{\theta} \xi_{\beta_{1} \ldots \beta_{q}}^{\sigma_{1} \ldots \sigma_{p}} & \delta_{\beta_{1}}^{\theta_{1}} \ldots \delta_{\beta_{q}}^{\theta_{q}} \delta_{\gamma_{1}}^{\sigma_{1}} \ldots \delta_{\gamma_{q}}^{\sigma_{p}}
\end{array}\right) \\
& =\left(\begin{array}{ccc}
\delta_{\alpha}^{\theta} & \partial_{\theta} \theta_{\alpha}-\partial_{\theta} \theta_{\alpha} & 0 \\
0 & \delta_{\theta}^{\alpha} & 0 \\
0 & \partial_{\theta} \xi_{\alpha_{1} \ldots \alpha_{q}}^{\sigma_{1} \ldots \sigma_{p}}-\partial_{\theta} \xi_{\alpha_{1} \ldots \alpha_{q}}^{\sigma_{1} \ldots \sigma_{p}} & \delta_{\alpha_{1}}^{\theta_{1}} \ldots \delta_{\alpha_{q}}^{\theta_{q}}
\end{array}\right)=\left(\begin{array}{ccc}
\delta_{\alpha}^{\theta} & 0 & 0 \\
0 & \delta_{\theta}^{\alpha} & 0 \\
0 & 0 & \delta_{\alpha}^{\theta}
\end{array}\right)=\delta_{C}^{A}=\widetilde{I} .
\end{aligned}
$$

Then we see from Theorem 5.2 that the complete lift ${ }^{c c} X$ of a vector field $X \in \Im_{0}^{1}\left(T^{*}\left(M_{n}\right)\right)$ has along $\beta_{\xi}\left(T^{*}\left(M_{n}\right)\right)$ components of the form

$$
{ }^{c c} X:\left(\begin{array}{l}
-L_{X} \theta \\
X \\
-L_{X} \xi
\end{array}\right)
$$


with respect to the adapted frame $\left\{B_{(\bar{\beta})}, C_{(\beta)}, C_{(\overline{\bar{\beta}})}\right\}$.

Let $A \in \Im_{q}^{p}\left(T^{*}\left(M_{n}\right)\right)$. If we take account of (2.1) and (5.5), we can easily prove that ${ }^{v v} A^{\prime}=\widetilde{A}\left({ }^{v v} A\right)$, where ${ }^{v v} A \in \Im_{0}^{1}\left(t_{q}^{p}\left(M_{n}\right)\right)$ is a vector field defined by

$$
{ }^{v v} A=\left(\begin{array}{l}
{ }^{v v} A^{\bar{\alpha}} \\
{ }^{v v} A^{\alpha} \\
{ }^{v v} A^{\overline{\bar{\alpha}}}
\end{array}\right)=\left(\begin{array}{l}
0 \\
0 \\
A_{\beta_{1} \ldots \beta_{q}}^{\alpha_{1} \ldots \alpha_{p}}
\end{array}\right),
$$

with respect to the adapted frame $\left\{B_{(\bar{\beta})}, C_{(\beta)}, C_{(\bar{\beta})}\right\}$ of $\beta_{\xi}\left(T^{*}\left(M_{n}\right)\right)$.

$B \omega, C X$ and $E \xi$ also have the components:

$$
B \omega=\left(\begin{array}{l}
\omega_{\alpha} \\
0 \\
0
\end{array}\right), C X=\left(\begin{array}{l}
0 \\
X^{\alpha} \\
0
\end{array}\right), E \xi=\left(\begin{array}{l}
0 \\
0 \\
\xi_{\beta_{1} \ldots \beta_{q}}^{\alpha_{1} \ldots \alpha_{p}}
\end{array}\right)
$$

respectively, with respect to the adapted frame $\left\{B_{(\bar{\beta})}, C_{(\beta)}, C_{(\bar{\beta})}\right\}$ of the cross-section $\beta_{\xi}\left(T^{*}\left(M_{n}\right)\right)$ determined by a tensor field $\xi$ of type $(p, q)$ in $T^{*}\left(M_{n}\right)$.

\section{References}

[1] Isham, C.J., Modern Differential Geometry for Physicists. World Scientific, 1999.

[2] Fattaev, H., The lifts of vector fields to the semitensor bundle of the Type (2, 0). Journal of Qafqaz University, 25 (2009), no. 1, $136-140$.

[3] Gezer, A., Salimov, A.A., Almost complex structures on the tensor bundles. Arab. J. Sci. Eng. Sect. A Sci., 33 (2008), no. 2, $283-296$.

[4] Husemoller, D., Fibre Bundles. Springer, New York, 1994.

[5] Lawson, H.B. and Michelsohn, M.L., Spin Geometry. Princeton University Press., Princeton, 1989.

[6] Ledger, A.J. and Yano, K., Almost complex structure on tensor bundles. J. Dif. Geom., 1 (1967), 355-368.

[7] Salimov, A., Tensor Operators and their Applications. Nova Science Publ., New York, 2013.

[8] Salimov, A.A. and Kadığlu, E., Lifts of derivations to the semitangent bundle. Turk J. Math., 24 (2000), 259-266.

[9] Steenrod, N., The Topology of Fibre Bundles. Princeton University Press., Princeton, 1951.

[10] Yano, K. and Ishihara, S., Tangent and Cotangent Bundles. Marcel Dekker, Inc., New York, 1973.

[11] Yıldırım, F., On a special class of semi-cotangent bundle, Proceedings of the Institute of Mathematics and Mechanics, (ANAS) 41 (2015), no. 1, 25-38.

[12] Yıldırım, F. and Salimov A., Semi-cotangent bundle and problems of lifts. Turk J. Math., 38 (2014), 325-339.

[13] Yildirım, F., Note on the cross-section in the semi-tensor bundle. New Trends in Mathematical Sciences, 5 (2017) , no. 2, $212-221$.

[14] Yıldırım, F., Asl, M.B. and Jabrailzade, F., Vector and affinor fields on cross-sections in the semi-cotangent bundle. Proceedings of the Institute of Mathematics and Mechanics, (ANAS) 43 (2017), no. 2, 305-315.

\section{Affiliations}

\section{FURKAN YILDIRIM}

ADDRESS: Atatürk University, Department of Mathematics, Faculty of Sci. Narman Vocational Training School, 25530, Erzurum Turkey.

E-MAIL: furkan.yildirim@atauni.edu.tr

ORCID ID : orcid.org/0000-0003-0081-7857 